American Journal of Pharmacology and Toxicology 5 (2): 80-88, 2010

ISSN 1557-4962

(C) 2010 Science Publications

\title{
Quercetin Liposomes via Nasal Administration Reduce Anxiety and Depression-Like Behaviors and Enhance Cognitive Performances in Rats
}

\author{
${ }^{1}$ Terdthai Tong-Un, ${ }^{1}$ Panakaporn Wannanon, \\ ${ }^{1}$ Jintanaporn Wattanathorn and ${ }^{2}$ Wathita Phachonpai \\ ${ }^{1}$ Department of Physiology, Faculty of Medicine, \\ ${ }^{2}$ Department of Physiology, Faculty of Medicine, Graduate School, \\ Faculty of Medicine, Khon Kaen University, Khon Kaen 40002, Thailand
}

\begin{abstract}
Problem statement: At present, the prevalence of neuropsychological disorders particularly anxiety, depression and dementia are increase. Many strategies have been developed in order to increase the efficiency of treatment and decrease side effects. Oxidative stress has been reported to play an important role on various path physiological states. Quercetin is a flavonoid commonly found in fruits and vegetables. It has potent antioxidant effects in vivo and in vitro. However, poor absorption, rapid metabolism and limited ability to cross the blood-brain-barrier are obstacles to its use for treatment of neuropsychological disorders. Nasal administration has been proposed as a noninvasive method to deliver bioactive substances to the brain. This route of administration has the potential of decreased hepatic metabolism and greater access to the brain tissue through the olfactory nerve compared to the oral route and could be a potential method of delivering quercetin to the CNS. Approach: This study was set up to determine the effect of quercetin liposomes via nasal administration on the anti-anxiety, anti-depression like activity and cognitive enhancing effect. In this case, Male Wistar rats were administered quercetin liposomes, containing $0.5 \mathrm{mg}$ of quercetin in $20 \mu \mathrm{L}$ (dose $=20 \mu \mathrm{g})$, via intranasal route once daily continually for 4 weeks. The anxiolytic activity, anti-depression like activity and cognitive enhancing effect were determined after single administration, 1-4 week of treatment using elevated plus maze test, forced swimming test and Morris water maze test respectively. Results: Quercetin liposomal encapsulation via nasal route possessed anxiolytic, anti-depression like activity and cognitive enhancing effect at all treatment duration. Conclusion: Our results demonstrated the anxiolytic, anti-depression like-activity and cognitive enhancing effect of quercetin liposomes via nasal administration and suggested that it might be used as a novel therapeutic strategy for mood disorder and cognitive deficit condition. However, further investigations about precise underlying mechanism are still required.
\end{abstract}

Key words: Quercetin liposomes, nasal administration, anxiety, anti-depression like activity, cognitive enhancing effect

\section{INTRODUCTION}

At present, it has been reported that more than 450 million people worldwide suffer from the mental and brain disorders, particularly anxiety, depression and dementia (Indian Council of Medical Research, 2001). Most of the drugs used nowadays have adverse side effects so the need for newer, better-tolerated and more efficacious treatments is remaining high.

Novel biological active compound from natural products appear to be the lead compounds which have been focused as the natural resource for drug development. Recently, oxidative stress has been reported to play an important role on various path physiological states (Golden et al., 2002). Therefore, the natural product possessing antioxidant has obtained very much attention.

Quercetin (3,5,7,3',4'-pentahydroxylflavone), a safe and dietary flavonoid generally found in vegetables and fruits such as onions, apples, tea and red wines (Formica and Regelson, 1995) and is of interest for nutraceutical and pharmaceutical uses (Bonina et al., 1996; Huang et al., 2006). It may exert beneficial health effects such as antivirus (Ohnishi and Bannai, 1993), certain forms of cancer (Lamson and Brignall, 2000), pulmonary and cardiovascular diseases (Middleton et al.,

Corresponding Author: Terdthai Tong-Un, Department of Physiology, Faculty of Medicine, Khon Kaen University, Khon Kaen 40002, Thailand Tel: 66-43-348394 
Am. J. Pharm. \& Toxicol., 5 (2): 80-88, 2010

2000) but also as neroprotection (Cho et al., 2006). Moreover, it is known as a strong free radical scavenger (Rodriguez et al., 2001). The idea of applying quercetin as the agent to reduce anxiety like, depression like activity and enhance cognition has become attractive and challenge. In order to apply quercetin for neuropsychological function, it is necessary to overcome the limitations of quercetin: Poor absorption and very low distribution to the brain after oral administration (Boer et al., 2005) due to both rapid metabolism (Manach et al., 1998) and difficulties in the penetration through the Blood-Brain Barrier (BBB) (Youdim et al., 2004). An effective dose of quercetin to exert certain neurological activities could be relatively high because of first-pass metabolism (Bhattaram et al., 2002).

Liposomes have been used as an effective delivery system to the brain, because the particles entrap the compounds and prevent rapid elimination or degradation as well as promote penetration through the BBB and distribution in the brain tissue (Krauze, 2006). Being entrapped in liposomes, a decrease in the dose of a compound to be administered is usually expected (Sharma and Sharma, 1997). The bilayer structure of liposomes mimics natural biomembranes. Also, liposomes formulations can be modified to serve the objectives.

Nasal administration offered many benefits including bypass the hepatic presystemic metabolism, simple dose adjustments, constant absorption and highly convenient for the patient administration (Wang et al., 2006). Moreover, nasal delivery was proposed to be the alternative administration route to target drugs directly to brain via the olfactory neurons (Illum, 2000; Mathison et al., 1998), therefore, this provides more opportunity for quercetin to enter the Central Nervous System (CNS) and then act on CNS to reduce anxiety, depression like activity and promote learning and memory with highly efficiency.

Based on all these points, the possible effects of quercetin liposomes via nasal administration on anxiolytic, anti-depression like activity and cognitive behaviors has been investigated.

\section{MATERIALS AND METHODS}

High-purity egg L- $\alpha$-phosphatidylcholine, type XVI-E (EPC), cholesterol (chol), quercetin dihydrate (98\% HPLC purity) and Polyethylene Glycol 400 (PEG) were purchased from Sigma (Barcelona, Spain). Other reagents used were analytical grade such as chloroform, ethanol and methanol (HPLC and analytical grade) from BDH Laboratory Supplies
(Poole, England), disodium hydrogen phosphate, perchloric acid and ortho phosphoric acids (Merck, Darmstadt, Germany), sodium dihydrogen phosphate (JT Baker Inc., Phillipsburg, New Jersey). All other chemicals were at least reagent grade and used as received.

Preparation of quercetin liposomes: Quercetin dehydrate (98\%), high-purity egg L- $\alpha$ phosphatidylcholine, Type XVI-E (EPC) and Cholesterol (CHOL) were prepared as quercetin liposomes. The method used was lipid thin film formation and extrusion (Guo et al., 2003; Liang et al., 2005; Zu et al., 2006).

Animals: Adult male Wistar rats $(180 \pm 20 \mathrm{~g}, 8$ weeks old) were obtained from National Animal Center, Salaya, Nakhon Pathom and they were housed in group of five per cage in standard metal cages at $22 \pm 2^{\circ} \mathrm{C}$ on 12:12 h light-dark cycle. All animals were given access to food and water ad libitum. The experiments were performed to minimize animal suffering in accordance with the internationally accepted principles for laboratory use and care of European Community (EEC directive of 1986; 86/609/EEC).

The experimental protocols were approved by the Institutional Animal Care and Use Committee.

Experimental protocol: In all part of this study, rats were divided into 4 groups of 8 each as described following except in the determination of stereotype behaviors.

Group 1: Naive intact control.

Group 2: Free liposomes: The rats in this group were administered free liposomes via nasal route.

Group 3: Liposomes + PEG: The rats in this group were administered PEG which used as vehicle of quercetin liposomes via nasal route.

Group 4: Liposomes + Quercetin (QCL): The rats in this group were administered quercetin liposomes via nasal route.

All animals were administered freshly prepared quercetin liposomes, containing $0.5 \mathrm{mg}$ of quercetin in $20 \mu \mathrm{L}$ (dose $=20 \mu \mathrm{g}$ ), being drawn into a micropipette, were administered to the right nasal cavity of each rat which was then holding still for $20 \mathrm{sec}$ to ensure the flow and contact of the quercetin liposomes to the nasal cavity whereas the control group received liposomes without quercetin or liposomes + PEG at the same volume once daily for 4 weeks. The rats were treated at lease $20 \mathrm{~min}$ prior to the daily experiment. 
Behaviors evaluation: The rats were divided into various groups as mentioned earlier. The behavioral profiles were assessed both after the single dose and repetitive administration of the substance as following: 1-4 week of treatment. All animals were submitted to the following behavior tasks (a) elevated plus maze test (b) Open field test (c) forced swimming test (d) cognitive function.

Elevated plus maze test: The elevated plus maze for rat consisted of open arms $(50 \times 10 \mathrm{~cm})$ and two enclosed arms $(50 \times 10 \mathrm{~cm})$ with $40 \mathrm{~cm}$ high walls, extending from a central platform $(10 \times 10 \mathrm{~cm})$. The arms were connected with a central square, $10 \times 10 \mathrm{~cm}$, to give the apparatus a plus sign appearance. The maze was raised to a height of $50 \mathrm{~cm}$ above floor. The maze floor and walls were constructed from dark opaque wood. Each rat was placed on the center of the platform facing an enclosed arm. Animals were tested individually and only once for $5 \mathrm{~min}$ according to the following parameters: Number of entries in the open and closed arms and time of performance in each of them (Lister, 1987). The time of performance measures the time spent by the animal in the opened and closed arms. The maze was cleaned following each trial to remove any residue or odors. Each rat was assessed individually $20 \mathrm{~min}$ after the treatment.

Open field test: In order to assure that anti-depression like activity and cognitive enhancing effect which determined by various tests just mentioned earlier were not false positive due to the effect of quercetin liposomes on motor behavior, we also determined the effect of quercetin liposomes on the spontaneous locomotor activity by open field test (Kulkarni and Dandiya, 1973). The open field apparatus was an arena $80 \mathrm{~cm}$ in diameter with a white, opaque wall $30 \mathrm{~cm}$ high. Rats were individually placed in the center of the arena and locomotor activity including the number of grooming, licking and rearing were scored within $5 \mathrm{~min}$.

Forced swimming test: In order to assess the antidepressant activity of plant extract, the modified Porsolt et al. (1978) test was conducted. In the first trial, the rats not yet treated were forced to swim in glass aquarium $(22 \mathrm{~cm}$ in diameter, $40 \mathrm{~cm}$ in height) containing $20 \mathrm{~cm}$ high fresh water at $25^{\circ} \mathrm{C}$ for $15 \mathrm{~min}$. Then, the assessments were performed after single, 14 week of treatment. During the test session, the immobility time was recorded by blind observer who has been trained for the observation. The rats were considered immobile when neither hind leg was moving; the rats were slightly hunched forward. The total duration of immobility was measured during the $5 \mathrm{~min}$ test. Upon removal from the water, rats were towel-dried and finally returned to their home cage.
Morris water maze test: The water maze consisted of a metal pool $(170 \mathrm{~cm}$ in diameter $\times 58 \mathrm{~cm}$ tall $)$ filled with tap water $\left(25^{\circ} \mathrm{C}, 40 \mathrm{~cm}\right.$ deep) divided into four quadrants. In the center of one quadrant was a removable escape platform below the water level and covered with a nontoxic milk powder. The pool was divided into four quadrants (NE, NW, SE and SW) by two imaginary lines crossing the center of the pool. For each animal, the location of invisible platform was placed at the center of one quadrant and remained there throughout training. The rats must memorize the platform location in relation to various environmental cues because there was nothing directly shows the location of the escape platform in and outside the pool. Therefore, the placement of the water tank and platform were the same in all acquisition trials. Each rat was gently placed in the water facing the wall of the pool from one of the four starting points $(\mathrm{N}, \mathrm{E}, \mathrm{S}$, or W) along the perimeter of the pool and the animal was allowed to swim until it found and climbed onto the platform. During training session, the subject was gently placed on the platform by the experimenter when it could not reach the platform in $60 \mathrm{sec}$. In either case, the subject was left on the platform for $15 \mathrm{sec}$ and removed from the pool. The time for animals to climb on the hidden platform was recorded as escape latency or acquisition time. In addition to the acquisition test, the determination of retention memory was carried out on the next day. According to this test, the platform was removed and the animals were placed into the water maze for $60 \mathrm{sec}$. The retention of memory or the time that the animal spent to swim around the previous location of platform before removing the platform on the test occurring in the next day was also recorded. It has been postulated that if the spatial memory of the rat for the trained platform location is accurate, the rat will swim to the platform location and search around the exact location. Therefore, the more accurate the spatial memory is, the greater the number of times rat swim across the trained platform. In each trial, the animal was quickly dried with towel before being returned to the cage. All test in Morris water maze tests were carried out within $30 \mathrm{~min}$ after the nasal administration of the substances.

Any enhancement of cognition would be reflected by a decrease in escape latency and increase in retention time.

Statistical analysis: Data are presented as Mean \pm Standard Error of Mean ( \pm SEM). One-way Analysis Of Variance (ANOVA), followed by Duncan's post hoc test. A probability level less than 0.05 was accepted as significance. 


\section{RESULTS}

Effect of quercetin liposomes on anxiolytic activity: To determine the effect of quercetin liposomes via nasal administration on anxiolytic activity, the results depicted in Fig. 1 and 2 showed that quercetin liposomes administered via nasal route produced significant $(\mathrm{p}<0.05)$ attenuate the diminution in both number of opened arms entries and time spent in the opened arms both after single and repetitive administration while both free liposomes and vehicle (PEG) failed to show the significant changes on both parameters mentioned above throughout the 4 weeks of treatment.

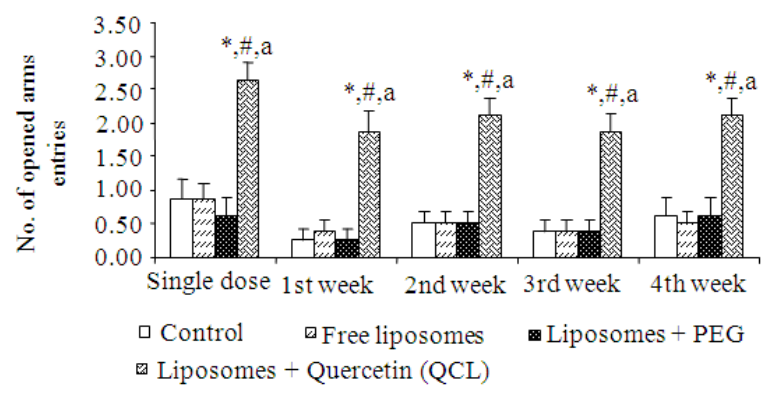

Fig. 1: Effect nasal administration of quercetin liposomes on the number of opened arms entries in the elevated plus maze test $(\mathrm{N}=8)$. Results were expressed as mean \pm SEM. ${ }^{* *}: \mathrm{p}-$ value $<0.05$ compared with control treated group; \#: p-value $<0.05$ compared with free liposomes treated group; ${ }^{\mathrm{a}}$ : $\mathrm{p}$-value $<0.05$ compared with liposomes + PEG treated group

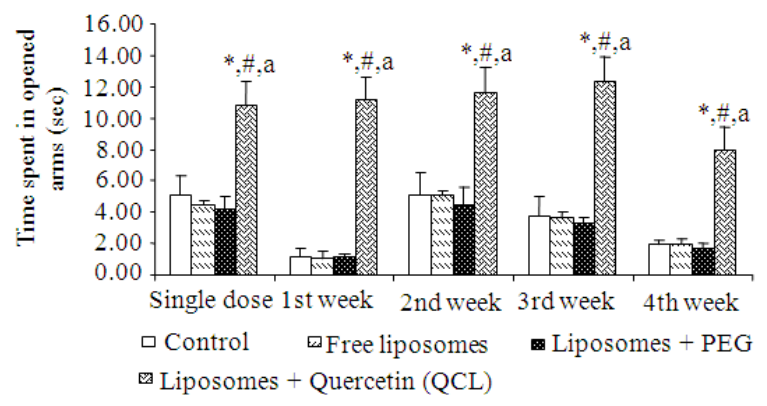

Fig. 2: Effect nasal administration of quercetin liposomes on the time spent in opened arms in the elevated plus maze test $(\mathrm{N}=8)$. Results are expressed as mean \pm SEM. ${ }^{*}:$ p-value $<0.05$ compared with control treated group; ": pvalue $<0.05$ compared with free liposomes treated group; ${ }^{\text {a: }}$ p-value $<0.05$ compared with liposomes + PEG treated group
Effect of quercetin liposomes on locomotor activity: The effects of quercetin liposomes via nasal administration on spontaneous motor behaviors including grooming, rearing and licking behaviors were demonstrated in Fig. 3-5. The results showed that the spontaneous behavior as mention earlier did not differ significantly between the control, vehicle treated group and quercetin liposomes throughout the experimental period.

Effect of quercetin liposomes on anti-depression activity: In order to investigate the anti-depressant effect of quercetin liposomes via nasal administration.

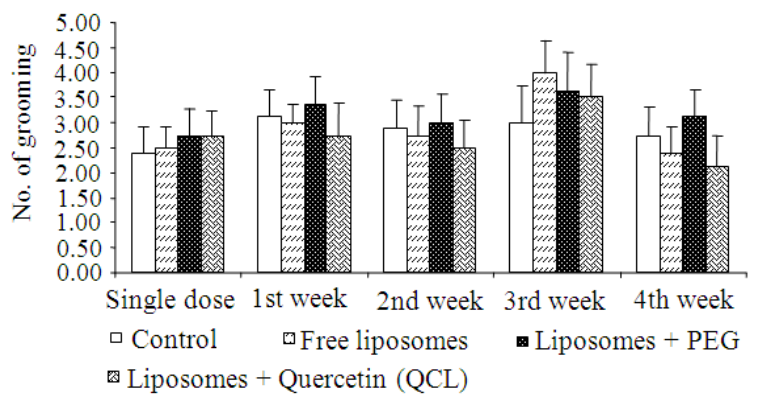

Fig. 3: Effect nasal administration of quercetin liposomes on grooming behaviors $(\mathrm{N}=8)$

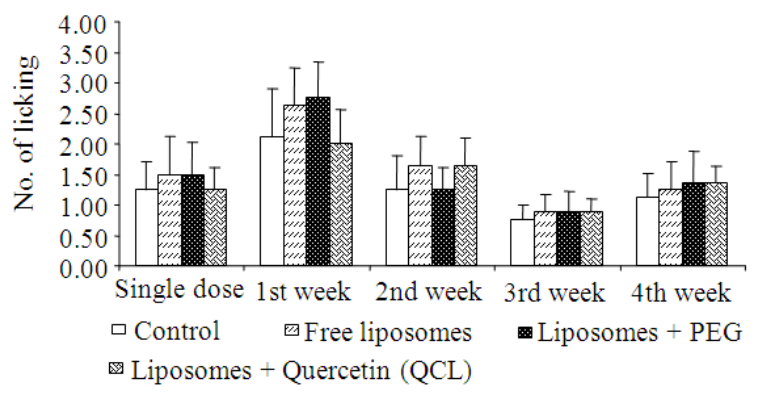

Fig. 4: Effect nasal administration of quercetin liposomes on licking behaviors $(\mathrm{N}=8)$

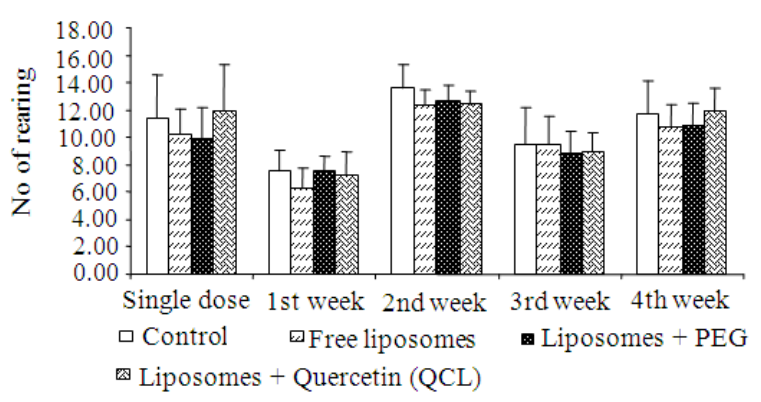

Fig. 5: Effect nasal administration of quercetin liposomes on rearing behaviors $(\mathrm{N}=8)$ 


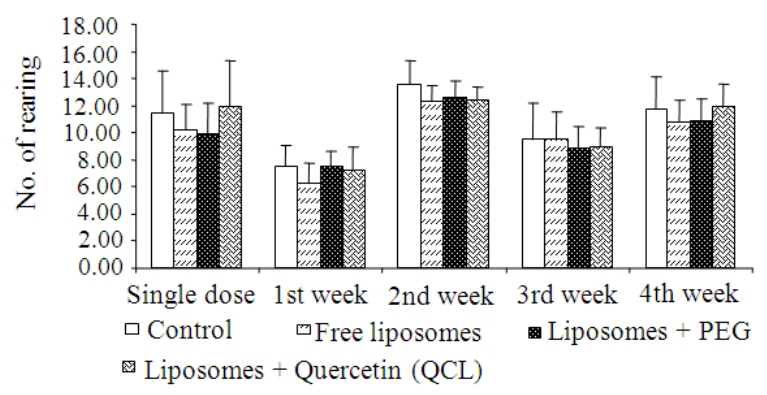

Fig. 6: Effect nasal administration of quercetin liposomes on anti-depression activity $(\mathrm{N}=8)$. Results are expressed as mean \pm SEM. ${ }^{*}:$ pvalue $<0.05$ compared with control treated group; \#: p-value $<0.05$ compared with free liposomes treated group; ${ }^{a}$ : $p$-value $<0.05$ compared with liposomes + PEG treated group

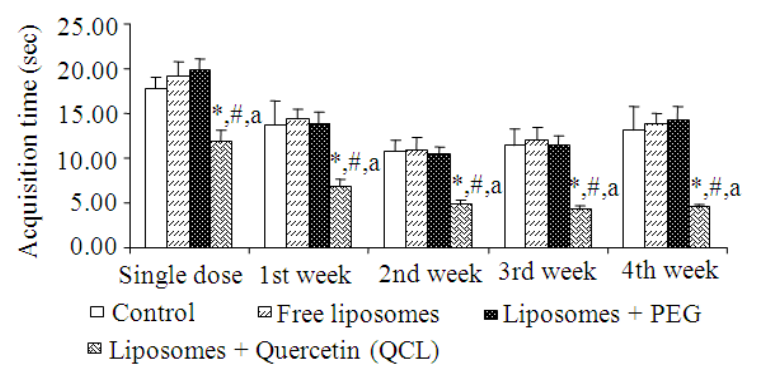

Fig. 7: Effect nasal administration of quercetin liposomes on the acquisition time of spatial learning in the Morris water maze test $(\mathrm{N}=8)$. Results were expressed as mean \pm SEM. ${ }^{*}:$ pvalue $<0.05$ compared with control treated group; \#: p-value $<0.05$ compared with free liposomes treated group; a: p-value $<0.05$ compared with liposomes + PEG treated group

The results showed that free liposomes and vehicle (PEG) treatment alone did not produce significant changes in immobility time at all treatment durations used in this study whereas quercetin liposomes produced a significant reduction in the immobility time at all treatment duration ( $\mathrm{p}<0.05$ all) as shown in Fig. 6.

Effect of quercetin liposomes on spatial memory: No significant changes in acquisition and retention time in control, vehicle (PEG) and free liposomes group rats. Surprisingly, Rats that received quercetin liposomes via nasal administration showed significantly decreased acquisition and increased retention time $(\mathrm{p}<0.05$ all $)$ as shown in Fig. 7 and 8 respectively, compared to control, free liposomes and vehicle. This indicates that nasal administration of quercetin liposomes could produce cognitive enhancing effect.

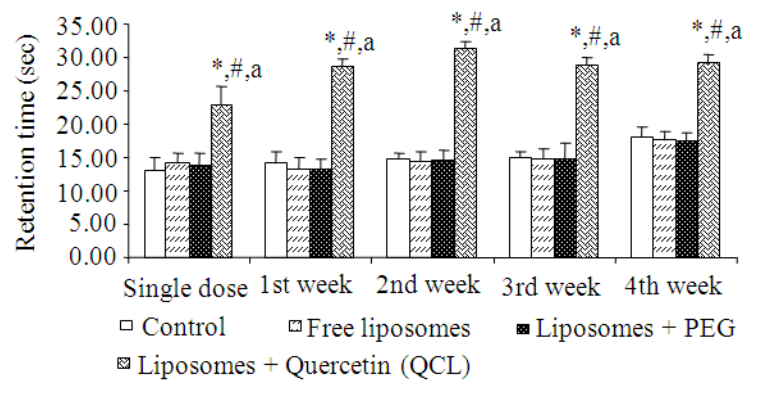

Fig. 8: Effect nasal administration of quercetin liposomes on the retention time of spatial learning in the Morris water maze test $(\mathrm{N}=8)$. Results were expressed as mean \pm SEM. ${ }^{*}: \mathrm{p}$ value $<0.05$ compared with control treated group; \#: p-value $<0.05$ compared with free liposomes treated group; a: p-value $<0.05$ compared with liposomes + PEG treated group

\section{DISCUSSION}

The current study demonstrate that nasal administration of quercetin liposomes, are effective in producing anxiolytic, anti-depression like activity and cognitive enhancing effect.

No unexpected mortality of any animals occurred after nasal administration of quercetin liposomes used in this study. As a result, vesicles (PEG) were considered to be safe at the dosing schedule used.

Forced Swimming Test (FST) is widely used for screening potential antidepressants (Borsini and Meli, 1988). The anti-depressant usually decreases immobility time without stimulating motor activity. Our forced swimming test data showed that the acute and the repeated administration of quercetin liposomes acted like an anti-depressant drug in rat, by decrease immobility time in FST.

Elevated Plus Maze test (EPM) is one of the most frequently used animal models in behavioral psychopharmacology for screening drugs with potential anxiolytic effects (Wall and Messier, 2000). In general, the reduction or increase in the number of entries and times spent into the open arms induced by a given substance had been regarded as good indicators of its anxiogenic or anxiolytic effect respectively (Pellow et al., 1985). The results of present study demonstrated that nasal administration of quercetin liposomes showed the anxiolytic-like effect in EPM.

To avoid false positive results in the FST and EPM, it is important to rule out the possibility that reductions in immobility time or increase in the number of entries and times spent into the open arms were not a result from psycho stimulant effects of the substance. 
In our study, either acute or repeated treatment with quercetin liposomes via nasal administration did not increase spontaneous motor activity at doses that produced an antidepressant-like effect and anxiolytic, indicating the specific effect of this substance on behavioral models predictive of antidepressant activity and anti-anxiety.

Previous studies proposed that there are at least two systems involved the depression including the monoaminergic and serotonergic system (Detke et al., 1995).

Monoamine Oxidase (MAOs) are enzymes which catalyses the oxidative domination of primary, secondary and tertiary amines (Edmondson et al., 2004). The primary functions of MAOs are the metabolism of exogenous amines and the regulation of neurotransmitter level and intracellular amine stores. Monoamine Oxidase A (MAO-A) preferentially deaminates serotonin (5-hydroxytryptamine) and nor epinephrine (Billett, 2004). Thus, inhibition of MAO-A may alleviate symptoms of depression.

Besides monoaminergic system, serotonergic system had long been implicated in the etiology of this condition. Decrease in brain concentrations of 5-HT and 5-HT1A (the major metabolite of 5-HT) were commonly observed in animals and in patients experiencing stress and depression, suggesting a dysfunction of serotonergic system (Southwick et al., 2005).

However, monoaminergic system and serotonergic system played the important role not only on antidepressant effect but also on anxiolytic effect (Aan Het Rot et al., 2009). Several lines of evidence demonstrated that numerous neurotransmitters including monoamine such as serotonin and nor epinephrine and GABA contributed the important role on anxiety. Previous studies reported that increase serotonin synthesis by the increasing the tryptophan supplement could improve social anxiety (Young and Gauthier, 1981). In addition, the level of norepinephrine and the activity of sympathetic nervous system were prolonged in patients with anxiety disorder (Sullivan et al., 1999). Moreover, most of the available anxiolytic drugs nowadays are still target on the GABAergic system (Ballenger, 1999).

Taken together, our results suggested that the mechanism underlying the anxiolytic and antidepression activity of quercetin liposomes via nasal administration may occur via the inhibition MAO-A, increase the serotonin level and increase in the GABAergic activity respectively.

These results are in accordance with the report of quercetin effect to inhibit MAO-A activity
(Chimenti et al., 2006). In addition, the electropharmacogram of adult rats which received quercetin have the same pattern as the well known antidepressant moclobemide, MAO-A inhibitor (Dimpfel, 2009). Moreover, quercetin could modify the function of GABA receptor (Goutman and Calvo, 2004). Therefore, the anti-depression and anxiolytic effects of quercetin liposomes might be associated with the ability to pass blood brain barrier of quercetin liposomes and its metabolites and exerted the influence to modify the function of monoamine and GABA as mentioned earlier.

Recently, it had been demonstrated that antidepressant, anti-anxiety which exerted its influence on serotonin could also exert the neuroprotective effect in hippocampus (Lucassen et al., 2004), a brain region which played important role in learning and memory (McNamara and Ronald, 1993). Previous studies in both humans and animals have demonstrated that the hippocampus is an important brain area for learning and memory particularly in the encoding as well as retrieval and long-term consolidation of spatial memory (Riedel et al., 2003). Therefore the present study also investigates the effect of queretin liposomes on the cognitive function. Our results showed that single and repetitive administration of quercetin liposomes administered via nasal route could improve spatial memory by decrease escape latency but increased retention time in Morris water maze test.

Numerous transmitters have been reported to play roles in spatial memory in Morris water maze test including acetylcholine and serotonin (Myhrer, 2003). The evidence stems from data of several authors demonstrated that there is a functional interaction between the cholinergic and serotonergic systems in the mediation of cognitive behavior (Steckler and Sahgal, 1995). In recent years, serotonin has been reported to play a crucial role in acquisition and consolidation process (Orsetti et al., 2003). Based on the role of serotonin on spatial memory in Morris water maze test and the effect of quercetin liposomes on serotonin as mentioned above, we do suggest that the cognitive enhancing effect of quercetin liposomes may probably occur partly via the facilitation of acquisition and consolidation process induced by the alteration in serotonin level. Moreover, serotonin also interacted with acetylcholine to regulate spatial memory (Cassel and Jeltsch, 1995). Therefore, we still cannot eliminate the effect of quercetin liposomes on acetylcholine. Taken all data together, the possible explanation for these changes might be related to the effect of quercetin liposomes on various regulators including the GABA, acetylcholine and monoamine system. 
Although, quercetin liposomes provided several advantages including the effective at very much low dose and the significant improvement in anxiolytic, anti-depression and cognitive enhancing effect were found very soon after single administration. However, further studies about the precise changes underlying these effects are still required.

\section{CONCLUSION}

Nasal administration of quercetin liposomes possesses anxiolytic, anti-depression like-activity and cognitive enhancing effect and suggested that it might be used as a novel therapeutic strategy for mood disorder and cognitive deficit condition.

\section{ACKNOWLEDGEMENT}

This work was partial supported by the National Nanotechnology Center (NANOTEC), NSTDA, National Science and Technology Development Agency, Thailand, through its program of Center of Excellence Network.

\section{REFERENCES}

Aan Het Rot, M., S.J. Mathew and D.S. Charney, 2009. Neurobiological mechanisms in major depressive disorder. Can. Med. Assoc. J., 180: 305-313. PMID: 19188629 DOI: 10.1503/cmaj.080697

Ballenger, J., 1999. Current treatments of the anxiety disorders in adults. Biol. Psychiat., 46: 1579-1594. DOI: 10.1016/S0006-3223(99)00220-6

Bhattaram, V.A., U. Graefe, C. Kohlert, M. Veit and H. Derendorf, 2002. Pharmacokinetics and bioavailability of herbal medicinal products. Phytomed., 9:1-33. DOI: 10.1078/1433-187X00210

Billett, E.E., 2004. Monoamine Oxidase (MAO) in human peripheral tissues. Neurotoxicol., 25: 139-148. PMID: 14697888

Boer, V.C.J., A.A. Dihal, H. van derWoude, I.C.W. Arts and S. Wolffram et al., 2005. Tissue distribution of quercetin in rats and pigs. J. Nutr., 135:1617-1618. PMID: 15987855

Bonina, F., M. Lanza, L. Montenegro, C. Puglisi and A. Tomaino et al., 1996. Flavonoids as potential agents against photo-oxidative skin damage. Int. J. Pharm., 145: 87-94. DOI: 10.1016/S03785173(96)04728-X

Borsini, F. and A. Meli, 1988. Is the forced swimming test a suitable model for revealing antidepressant activity. Psychopharmacol. Berl., 94: 147-160. PMID: 3127840
Cassel, J.C. and H. Jeltsch, 1995. Serotonergic modulation of cholinergic function in the central nervous system: Cognitive implications. Neuroscience, 69: 1-41. PMID: 8637608

Chimenti, F., F. Cottiglia, L. Bonsignore, L. Casu and M. Casu et al., 2006. Quercetin as the active principle of Hypericum hircinum exerts a selective inhibitory activity against MAO-A: Extraction, biological analysis and computational study. J. Nat. Prod., 69: 945-949. PMID: 16792415

Cho, J.Y., I.S. Kim, Y.H. Jang, A.R. Kim and S.R. Lee, 2006. Protective effect of quercetin, a natural flavonoid against neuronal damage after transient global cerebral ischemia. Neurosci. Lett., 404: 330-335. PMID: 16806698

Detke, M., M. Rickels and I. Lucki, 1995. Active behaviors in the rat forced swimming test differentially activated by serotonergic and noradrenergic antidepressants. Psychopharmacol., 121: 66-72. PMID: 8539342

Dimpfel, W., 2009. Rat electropharmacograms of the flavonoids rutin and quercetin in comparison to those of moclobemide and clinically used reference drugs suggest antidepressive and/or neuroprotective action. Phytomed, 16: 287-294. PMID: 19303757

Edmondson, D.E., A. Mattevi, C. Binda, M. Li and F. Hubalek, 2004. Structure and mechanism of monoamine oxidase. Curr. Med. Chem., 11: 1983-1993. PMID: 15279562

Formica, J.V. and W. Regelson, 1995. Review of the biology of quercetin and related bioflavonoids. Food. Chem. Toxicol., 33: 1061-80. PMID: 8847003

Golden, T.R., D.A. Hinerfeld and S. Melov, 2002. Oxidative stress and ageing: Beyond correlation. Aging Cell 1: 117-123. PMID: 12882341

Goutman, J.D. and D.J. Calvo, 2004. Studies on the mechanisms of action of picrotoxin, quercetin and pregnanolone at the GABAp1 receptor. Br. J. Pharmacol., 141: 717-727. PMID: 14732759

Guo, J., Q. Ping, G. Jiang, L. Huang and Y. Tong, 2003. Chitosan-coated liposomes: Characterization and interaction with leuprolide. Int. J. Pharm., 260: $\quad 167-173 . \quad$ DOI: $10.1016 /$ S03785173(03)00254-0

Huang, S.L., C.L. Hsu and G.C. Yen, 2006. Growth inhibitory effect of quercetin on SW 872 human liposarcoma cells. Life Sci., 79: 203-209. DOI: 10.1016/j.lfs.2005.12.046

Indian Council of Medical Research, (ICMR), 2001. Developments in mental health scenario: Need to stop exclusion-dare to care. ICMR Bull., 31: 1-9. http://www.emro.who.int/MNH/WHD/WHDBrochure.pdf 
Illum, L., 2000 Transport of drugs from the nasal cavity to the central nervous system. Eur. J. Pharm. Sci., 11: 1-18. PMID: 109137

Krauze, L., 2006. Real-time imaging and quantification of brain delivery of liposomes. Pharm. Res., 23: 2493-2504. PMID: 16972184

Kulkarni, S.K. and P.C. Dandiya, 1973. Effects of antidepressant agents on open field behavior in rats. Psychopharmacologia, 33: 333-338. PMID: 4798172

Lamson, D.W. and M.S. Brignall, 2000. Antioxidants and cancer. Part III. Quercetin. Altern. Med., 5: 196-208. PMID: 10869101

Liang, M.T., N.M. Davies and I. Toth, 2005. Encapsulation of lipopeptides within liposomes: Effect of number of lipid chains, chain length and method of liposome preparation. Int. J. Pharm., 301: 247-254. PMID: 16054787 DOI: 10.1016/j.ijpharm.2005.06.010

Lister, R.G., 1987. The used of a plus-maze to measure anxiety in the mouse. Psychopharmacology, 92: 180-185. PMID: 3110839

Lucassen, P.J., E. Fuchs and B. Czeh, 2004. Antidepressant treatment with tianeptine reduces apoptosis in the hippocampal dentate gyrus and temporal cortex. Biol. Psychiat., 55: 789-796. PMID: 15050859

Manach, C., C. Morand, V. Crespy, C. Demignee and O. Texier et al., 1998. Quercetin is recovered in human plasma as conjugated derivatives which retain antioxidant properties. FEBS Lett., 426: 331-336. PMID: 9600261

Mathison, S., R. Nagilla and U.B. Kompella, 1998. Nasal route for direct delivery of solutes to the central nervous system: Factor fiction. J. Drug Target., 5: 415-441. PMID: 9783675

McNamara, R.K. and W.S. Ronald, 1993. The neuropharmacological and neurochemical basis of place learning in the Morris water maze. Brain Res. Rev. 18: 33-49. PMID: 8467349

Middleton, E., C. Kandaswami and T.C. Theoharides, 2000. The effects of plant flavonoids on mammalian cells: Implications for inflammation, heart disease and cancer. Pharm. Rev., 52: 673-751. PMID: 11121513

Myhrer, T., 2003. Neurotransmitter systems involved in learning and memory in the rat: A meta-analysis based on studies of four behavioral tasks. Brain Res. Rev., 41: 268-287. PMID: 12663083

Ohnishi, E. and H. Bannai, 1993. Quercetin potentiates TNF-induced antiviral activity. Antiv. Res., 22: 327-331. PMID: 8279819
Orsetti, M., A. Dellarole, S. Ferri and P. Ghi, 2003. Acquisition, retention and recall of memory after injection of RS67333, a 5-HT (4) receptor agonist, into the nucleus basalis magnocellularis of the rat. Learn. Mem., 10: 420-426. PMID: 14557615

Pellow, S., P. Chopin, S.E. File and M. Briley, 1985. Validation of open: Closed arm entries in an elevated plus-maze as a measure of anxiety in the rat. J. Neurosci. Methods, 14: 149-167. DOI: 10.1016/0165-0270(85)90031-7

Porsolt, R.D., G. Anton, N. Blavet and M. Jalfre, 1978. Behavioral despair in rats: A new model sensitive to antidepressant treatments. Eur. J. Pharmacol., 47: 379-391. PMID: 204499

Riedel, G., B. Platt and J. Micheau, 2003. Glutamate receptor function in learning and memory. Behav. Brain Res., 140: 1-47. PMID: 12644276

Rodriguez, R.J., C.L. Miranda, J.F. Stevens, M.L. Deinzer and D.R. Buhler, 2001. Influence of prenylated and non-prenylated flavonoids on liver microsomal lipid peroxidation and oxidative injury in rat hepatocytes. Food Chem. Toxicol., 39: 437-445. PMID: 11313109

Sharma, A. and U.S. Sharma, 1997. Liposomes in drug delivery: Process and limitations. Int. J. Pharm., 154:123-140. DOI: 10.1016/S0378-5173(97)00135-X

Southwick, S.M., M. Vythilingam and D.S. Charney, 2005. The psychobiology of depression and resilience to stress: Implications for prevention and treatment. Annu. Rev. Clin. Psychol., 1: 255-291. PMID: 17716089

Steckler, T. and A. Sahgal, 1995. The role of serotoninergic-cholinergic interactions in the mediation of cognitive behavior. Behav. Brain Res., 67: 165-199. PMID: 7779290

Sullivan, G., J. Coplan, J. Kent and J. Gorman, 1999. The noradrenergic system in pathological anxiety: A focus on panic with relevance to generalized anxiety and phobia. Biol. Psychiat., 46: 1205-1218. PMID: 10560026

Wall, P.M. and C. Messier, 2000. Ethological confirmatory factor analysis of anxiety-like behavior in the murine elevated plus-maze. Behav. Brain. Res., 114: 199-212. PMID: 10996061

Wang, X., H. He, W. Leng and X. Tang, 2006. Evaluation of brain-targeting for the nasal delivery of estradiol by the microdialysis method. Int. J. Pharm., 317: 40-46. DOI: 10.1016/j.ijpharm.2006.02.055

Youdim, K.A., M.Z. Qaiser, D.J. Begley, C.A. RiceEvans and N.J. Abbott, 2004. Flavonoid permeability across an in situ model of the bloodbrain barrier. Free. Radic. Biol. Med., 36: 592-604. PMID: 14980703 
Young, S. and S. Gauthier, 1981. Effect of tryptophan administration on tryptophan, 5hydroxyindoleacetic acidand indoleacetic acid in human lumbar and cisternal cerebrospinal fluid. J. Neurol. Neurosurg. Psychiat., 44: 323-327. PMID: 6165809
Zu, Y., C. Li, Y. Fu and C. Zhao, 2006. Simultaneous determination of catechin, rutin, quercetin kaempferol and isorhamnetin in the extract of sea buckthorn (Hippophae rhamnoides L.) leaves by RP-HPLC with DAD. J. Pharm. Biom. Anal., 41: 714-719. PMID: 16520013 